\title{
Urbano y rural: diferencias en la alimentación de los hogares argentinos según nivel de ingreso y área de residencia
}

\author{
Urban and rural: differences in the diet of Argentine \\ households according to income level and area of \\ residence
}

María Elisa Zapata ${ }^{1}$, Alicia Rovirosa², Esteban Carmuega ${ }^{3}$

${ }^{1}$ Magíster en Nutrición Humana y Calidad de los Alimentos. Investigadora adjunta, Centro de Estudios sobre Nutrición Infantil Dr. Alejandro O'Donnell (CESNI), Ciudad Autónoma de Buenos Aires, Argentina. $\checkmark$ (iD)

${ }^{2}$ Bioquímica. Investigadora adjunta, Centro de Estudios sobre Nutrición Infantil Dr. Alejandro O'Donnell (CESNI), Ciudad Autónoma de Buenos Aires, Argentina. $\triangle$ (iD)

${ }^{3}$ Médico Pediatra. Director, Centro de Estudios sobre Nutrición Infantil Dr. Alejandro O'Donnell (CESNI), Ciudad Autónoma de Buenos Aires, Argentina. $\triangle$ iD

\begin{abstract}
RESUMEN El entorno y el nivel socioeconómico son determinantes del comportamiento alimentario porque inciden en la disponibilidad, la accesibilidad y las preferencias alimentarias. Con el objetivo de describir el consumo aparente de alimentos y la disponibilidad de energía y nutrientes de hogares urbanos y rurales de la Argentina, según su nivel de ingresos, se analizó la Encuesta Nacional de Gastos de los Hogares 2004-2005. Se calculó el consumo aparente promedio de alimentos y bebidas en gramos o mililitros de peso neto por adulto equivalente por día, de hogares urbanos y rurales, y según quintil de ingresos per cápita del hogar. El 7\% fueron hogares rurales, y presentaron mayor proporción de familias con bajos ingresos respecto a los urbanos. Existe un patrón de consumo aparente de alimentos y bebidas distinto entre hogares rurales y urbanos de Argentina, y además existen diferencias entre los hogares según el nivel de ingresos en ambos entornos. Conocer los contrastes y su magnitud es de gran utilidad para buscar estrategias tendientes a mejorar la alimentación de la población.
\end{abstract}

PALABRAS CLAVES Población Rural; Alimentos y Bebidas; Ingreso Familiar; Encuestas Nutricionales; Argentina.

\begin{abstract}
The environment and the socioeconomic level are determinants of eating behavior because they affect availability, accessibility and food preferences. In order to describe the apparent consumption of food and the availability of energy and nutrients in urban and rural households in Argentina according to their income level, the 20042005 National Household Expenditure Survey was analyzed. The average apparent consumption of food and beverages was calculated in grams or milliliters of net weight per adult equivalent per day, for urban and rural households, and by household income per capita quintiles. Rural households made up $7 \%$ of the sample, and had a higher proportion of low-income families than urban households. There is different pattern of apparent consumption of food and beverages among rural and urban households in Argentina, and there are also differences between households according to the level of income in both environments. Knowing the content and magnitude of these contrasts is of great use in looking for strategies to improve the population's diet.
\end{abstract}

KEY WORDS Rural Population; Food and Beverages; Family Income; Nutrition Surveys; Argentina. 


\section{INTRODUCCIÓN}

A nivel mundial, más cantidad de personas viven en áreas urbanas que en áreas rurales. En 1950, el 30\% de la población mundial era urbana; en 2014 , el $54 \%$ y, para 2050 , se proyecta que el $66 \%$ de la población mundial será urbana ${ }^{(1)}$. En Argentina, el proceso de urbanización de la población comenzó muy temprano en la historia del país. Como resultado del desplazamiento de la población hacia áreas urbanas, la población rural disminuyó, a lo largo del siglo XX, tanto en términos absolutos como relativos: en el censo nacional del año 1895 se registró un $65 \%$ de población rural ${ }^{(2)} y$, en 1914 , el $47 \%$; en el censo de 1970 descendió al 21\%(3); en 1991, el $13 \%$ de los habitantes residía en localidades de menos de 2.000 habitantes o disperso en campo abierto ${ }^{(4)}$; y el censo de 2010 relevó que el $9 \%$ de la población residía en áreas rurales ${ }^{(5)}$. Este descenso se produjo de forma heterogénea, las provincias y las regiones experimentaron un proceso diferente de evolución de la población rural siguiendo una compleja trama de factores ambientales, económicos, sociales y políticos ${ }^{(2)}$.

La vida urbana a menudo se asocia con niveles más altos de alfabetización y educación, mejor sistema de salud, mayor acceso a servicios sociales y mejores oportunidades para la participación cultural y política. Sin embargo, el crecimiento urbano rápido y no planificado amenaza el desarrollo sostenible cuando no se desarrolla la infraestructura necesaria o cuando no se implementan políticas para garantizar que los servicios de las ciudades se distribuyan equitativamente ${ }^{(1)}$.

Tanto el lugar de residencia como el contexto socioeconómico ejercen influencia sobre las elecciones y comportamientos alimentarios, porque modifican la disponibilidad y la accesibilidad ${ }^{(6)}$ y por las diferentes costumbres y hábitos propios del entorno. Además, los procesos demográficos, tecnológicos, económicos y ambientales que ocurren simultáneamente en todo el mundo están teniendo un impacto en el suministro de alimentos. Estos cambios, junto con la rápida urbanización y globalización, ejercen un efecto sobre la alimentación, con una tendencia hacia una mayor preferencia por los alimentos manufacturados y listos para comer $^{(7,8,9,10)}$. En las últimas décadas hubo un interés creciente en comprender cómo el ambiente afecta la salud de la población. En particular, por saber cómo los entornos alimentarios del lugar de residencia, los barrios, las escuelas y los lugares de trabajo condicionan la elección de alimentos y, en última instancia, el riesgo poblacional de obesidad y otras enfermedades crónicas relacionadas con la alimentación $\mathrm{n}^{(11,12,13)}$; entendiendo el entorno alimentario como el conjunto de factores físicos, sociales, económicos, culturales y políticos que afectan la accesibilidad, disponibilidad y adecuación de los alimentos dentro de una comunidad o región ${ }^{(14,15)}$.

La escasez de estudios poblacionales con representatividad nacional, que utilicen métodos de evaluación alimentaria considerados como patrón de referencia, como recordatorios de 24 horas o registros de consumo de alimentos, ha sido durante mucho tiempo un obstáculo para realizar nutrición basada en la evidencia y planificar políticas alimentarias. Las encuestas de gastos de hogares se realizan en forma periódica, tienen representatividad nacional y se han utilizado en los últimos años en muchos países como una medida aproximada para superar la brecha sobre información alimentaria y nutricional ${ }^{(16,17,18)}$. En Argentina, se han realizado tres Encuestas Nacionales de Gasto de los Hogares (ENGHo) a nivel nacional, y en la de 2004-2005 se incluyó población rural ${ }^{(19)}$. Dado que no hemos hallado publicaciones que evalúen, a nivel nacional, las diferencias en el consumo alimentario y la ingesta de nutrientes de la población procedente de zonas urbanas y rurales según los ingresos del hogar, el objetivo de este trabajo fue describir el consumo aparente de alimentos y la disponibilidad de energía y nutrientes de hogares urbanos y rurales de la Argentina según su nivel de ingreso. 


\section{MATERIAL Y METODO}

El análisis se realizó a partir de la información recolectada por la Encuesta Nacional de Gastos de los Hogares (ENGHo) del año 20042005 conducida por el Instituto Nacional de Estadísticas y Censos (INDEC). La encuesta tomó una muestra probabilística, polietápica y estratificada de 29.138 hogares. De acuerdo a lo definido por el INDEC para esta encuesta, se denomina rural a la población dispersa en campo abierto sin constituir centros poblados, mientras que urbano refiere a la población que reside en áreas de 2.000 o más habitantes e incluye la población rural agrupada $^{(20)}$.

La información fue recolectada entre octubre de 2004 y diciembre de 2005. Los hogares seleccionados registraron sus ingresos mensuales y sus gastos durante el periodo de una semana. Con relación al ingreso, el cuestionario incluye las entradas de dinero que percibieron cada uno de los miembros del hogar en los seis meses anteriores a la semana de la encuesta y, con relación a los gastos, los participantes deben registrar las adquisiciones en las siguientes categorías: alimentos y bebidas; indumentaria y calzado; propiedades, combustibles, agua y electricidad; equipamiento y mantenimiento del hogar; salud; transporte y comunicaciones; esparcimiento; enseñanza; y bienes y servicios para el cuidado personal. Se consideran los gastos de consumo de bienes y servicios al contado o a crédito, como también los bienes primarios producidos por el hogar para su propio consumo como los alimentos producidos en huerta y/o corral.

Para el análisis se utilizaron los datos de consumo de alimentos y bebidas e ingresos de los hogares. El consumo aparente o disponibilidad para consumo, que corresponde a la estimación del consumo a partir de los datos de compra de alimentos ${ }^{(21)}$, fue calculado a partir de la cantidad de alimentos y bebidas adquiridos para consumir en el hogar o comprados y consumidos fuera del hogar (en restaurantes, bar y comedores), recolectados mediante un cuestionario que incluyó 400 ítems. Los alimentos y bebidas registrados en el cuestionario por cada hogar fueron transformados de peso bruto a gramos de peso neto mediante el factor de corrección correspondiente a cada alimento del software SARA ${ }^{(22)}$ y de la tabla recopilada por la Escuela de Nutrición de la Universidad de Buenos Aires ${ }^{(23)}$. En el caso de alimentos y bebidas registrados como unidades, se transformaron en gramos de peso neto según los valores de la tabla de pesos y medidas de referencia del software SARA ${ }^{(22)}$ y la tabla compilada por López ${ }^{(23)}$. Para los alimentos listos para consumir, comprados y consumidos dentro o fuera del hogar, se consideró el peso de la porción lista para consumo de sándwiches, tartas, empanadas, pizza, entre otros. En el caso de desayuno o merienda se asignó el valor correspondiente al volumen de una taza de infusión y dos facturas o tostadas untadas y, para almuerzo y cena, el peso promedio de un plato elaborado y un vaso de bebida. Las leches en polvo, jugos en polvo e infusiones se presentan en valores reconstituidos, según dilución estándar indicada por el fabricante.

Luego de la transformación de todos los alimentos y bebidas a gramos o mililitros de peso neto por día, las cantidades totales fueron divididas por el total de adultos equivalentes en el hogar. Considerando que un adulto equivalente con coeficiente 1 corresponde a un varón de entre 30 y 59 años, con una actividad moderada y necesidades energéticas de $2.700 \mathrm{kcal}$, cada individuo del hogar tiene su coeficiente de acuerdo a las necesidades calóricas de su grupo biológico ${ }^{(19)}$. El consumo aparente se expresa en peso neto como gramos o mililitros promedio de alimentos y bebidas por adulto equivalente por día, para hogares urbanos y rurales, y según quintil de ingresos per cápita del hogar. Para presentar los resultados, los alimentos y bebidas se agruparon de acuerdo a sus características nutricionales considerando los grupos de alimentos de las Guías alimentarias para la población argentina ${ }^{(24,25)}$.

La energía y nutrientes fueron calculados a partir del promedio de peso neto de alimentos y bebidas por adulto equivalente por 
día, de acuerdo a la tablas de composición nutricional de Argenfoods $^{(26)}$, del software SARA, de la base de datos de United States Department of Agriculture (USDA) ${ }^{(27)}$ y de datos analizados en el laboratorio del Centro de Estudios sobre Nutrición Infantil (CESNI).

Se calculó el quintil de ingreso a partir del dato de ingreso del hogar per cápita. Tanto el consumo aparente de alimentos, como la disponibilidad de energía y nutrientes, se presentan estratificados según área de residencia (urbano y rural) y por quintil de ingreso per cápita de cada hogar.

El análisis estadístico descriptivo fue realizado mediante Microsoft Excel ${ }^{\circledR}$ y SPSS ${ }^{\circledR}$ versión 20.0 (SPSS Inc., Chicago, IL, USA), se consideró el factor de ponderación correspondiente a cada hogar.

\section{RESULTADOS}

Los hogares rurales fueron el $7 \%$ de la muestra, con diferente proporción en cada región del país. En el Noreste (NEA) el 18\%, en Cuyo el $14 \%$, en el Noroeste (NOA) el $12 \%$, en la región Pampeana el $8 \%$, en la Patagonia el 5\%, y ninguno en Gran Buenos Aires. La Tabla 1 muestra la cantidad de hogares e individuos encuestados en el área urbana y rural, y sus características. La cantidad de individuos por hogar en área rural es levemente superior y se observa una mayor proporción de jefes de hogar de sexo masculino, con menor proporción de cobertura médica y menor nivel de instrucción. Mientras que $19 \%$ de los hogares urbanos se ubican en el quintil 1 de ingresos en los hogares rurales la cifra asciende al $34 \%$, y la proporción de hogares rurales en el $5^{\circ}$ quintil de ingresos es la mitad respecto a los urbanos. La disponibilidad de huerta o corral para consumo del hogar es muy superior en los hogares rurales.

Los hogares rurales tienen mayor consumo aparente de gran parte de los grupos de alimentos. El consumo de cereales es 2,5 veces mayor (urbanos $=93 \mathrm{~g}$, rurales $=232 \mathrm{~g}$ ) $y$, del total de cereales consumidos en los hogares rurales, la mitad corresponde a la harina de trigo. Las legumbres presentan muy bajo consumo $(<5 \mathrm{~g} / \mathrm{d})$ con cifras discretamente más altas en los hogares rurales. La misma tendencia se observa en las hortalizas feculentas $\quad$ (urbanos $=99 \mathrm{~g}$, rurales $=122 \mathrm{~g}$ ), el huevo (urbanos $=20 \mathrm{~g}$, rurales $=25 \mathrm{~g}$ ), los aceites y grasas (urbanos $=37 \mathrm{~g}$, rurales $=55 \mathrm{~g}$ ) especialmente, por los aceites, tanto mezcla como puro, por las grasas animales y los azúcares y dulces (urbanos $=61 \mathrm{~g}$, rurales $=96 \mathrm{~g}$ ) donde el azúcar es el principal responsable de

\begin{tabular}{|c|c|c|}
\hline Caracteristicas de los hogares & Entorno urbano & Entorno rural \\
\hline Número de hogares & 26.968 & 2.170 \\
\hline Número de hogares (ponderado) & 10.472 .087 & 740.295 \\
\hline Número de individuos & 96.064 & 8.794 \\
\hline Número individuos (ponderado) & 35.409 .909 & 2.848 .068 \\
\hline \multicolumn{3}{|l|}{$\begin{array}{l}\text { Promedio de miembros por hogar según } \\
\text { grupos de edad }\end{array}$} \\
\hline$<6$ años & $0,3 \pm 0,7$ & $0,5 \pm 0,8$ \\
\hline 6 a 17 años & $0,7 \pm 1,1$ & $1,0 \pm 1,4$ \\
\hline 18 a 64 años & $2,0 \pm 1,2$ & $2,0 \pm 1,2$ \\
\hline 65 años y más & $0,3 \pm 0,6$ & $0,3 \pm 0,6$ \\
\hline Total & $3,4 \pm 1,9$ & $3,8 \pm 2,2$ \\
\hline Adultos equivalentes & $2,7 \pm 1,5$ & $3,1 \pm 1,7$ \\
\hline $\begin{array}{l}\text { Porcentaje de hogares con jefe de hogar } \\
\text { hombre }\end{array}$ & $69,5 \%$ & $86,0 \%$ \\
\hline $\begin{array}{l}\text { Porcentaje de hogares con jefe de hogar con } \\
\text { cobertura médica }\end{array}$ & $68,4 \%$ & $51,4 \%$ \\
\hline \multicolumn{3}{|l|}{$\begin{array}{l}\text { Porcentaje de hogares según nivel de } \\
\text { instrucción del jefe de hogar }\end{array}$} \\
\hline Sin instrucción o con primario incompleto & $16,3 \%$ & $43,6 \%$ \\
\hline Primario completo & $44,5 \%$ & $46,4 \%$ \\
\hline Secundario completo & $19,0 \%$ & $6,5 \%$ \\
\hline Terciario o superior & $19,9 \%$ & $3,2 \%$ \\
\hline Educación especial & $0,1 \%$ & $0,0 \%$ \\
\hline \multicolumn{3}{|l|}{$\begin{array}{l}\text { Porcentaje de hogares según quintil de } \\
\text { ingreso }\end{array}$} \\
\hline Quintil 1 & $19,0 \%$ & $34,3 \%$ \\
\hline Quintil 2 & $19,8 \%$ & $22,4 \%$ \\
\hline Quintil 3 & $20,2 \%$ & $16,7 \%$ \\
\hline Quintil 4 & $20,4 \%$ & $14,7 \%$ \\
\hline Quintil 5 & $20,6 \%$ & $11,9 \%$ \\
\hline $\begin{array}{l}\text { Porcentaje de hogares con disponibilidad de } \\
\text { corral para consumo }\end{array}$ & $2,8 \%$ & $46,1 \%$ \\
\hline $\begin{array}{l}\text { Porcentaje de hogares con disponibilidad de } \\
\text { huerta para consumo del hogar }\end{array}$ & $4,2 \%$ & $32,4 \%$ \\
\hline
\end{tabular}


las diferencias (urbanos $=35 \mathrm{~g}$, rurales $=80 \mathrm{~g}$ ). Para algunos grupos de alimentos las diferencias son pequeñas, como es el caso de los panificados y galletitas (urbanos $=198 \mathrm{~g}$, rurales $=193 \mathrm{~g}$ ), la leche (urbanos $=138 \mathrm{ml}$, rurales $=132 \mathrm{ml}$ ) y las carnes (urbanos $=187 \mathrm{~g}$, rurales $=204 \mathrm{~g}$ ), donde además las diferencias residen en el tipo de leche y carne. En los hogares rurales se observa un consumo del doble de leche en polvo y 8,6 veces más alto carne ovina y porcina (urbanos $=3 \mathrm{~g}$, rurales $=22 \mathrm{~g}$ ) pero la mitad de pescado (urbanos $=8 \mathrm{~g}$, rurales $=4 \mathrm{~g}$ ).

En todos los grupos de alimentos mencionados, el consumo tiene relación directa con los ingresos del hogar, a excepción de los panificados y galletitas que, en hogares urbanos, la cantidad total no varía según los ingresos, los cereales que disminuyen cuando crecen los ingresos en los hogares rurales y la papa que se consume en cantidad similar entre quintiles en ambos entornos (Tabla 2). Los únicos grupos de alimentos que se consumen en menor cantidad en hogares rurales son:
- frutas (urbanos $=107 \mathrm{~g}$, rurales $=75 \mathrm{~g}$ ),

- hortalizas no feculentas (urbanos $=144 \mathrm{~g}$, rurales $=134 \mathrm{~g}$ ),

- yogur (urbanos $=32 \mathrm{~g}$, rurales15g),

- quesos (urbanos $=23 \mathrm{~g}$, rurales $=11 \mathrm{~g}$ ),

- comidas listas para comer (urbanos $=89 \mathrm{~g}$, rurales $=35 \mathrm{~g}$ ),

- gaseosas y jugos (urbanos $=310 \mathrm{ml}$, rurales $=230 \mathrm{ml}$ ).

Para estos alimentos y grupos de alimentos, el consumo aumenta a medida que crecen los ingresos, a excepción de las gaseosas y jugos donde la diferencia entre los hogares con diferentes ingresos es menor (Tabla 2).

Al comparar según el entorno en el que se ubica el hogar a igual quintil de ingreso, se observa que, en el quintil 1, los hogares rurales consumen más harina y cereales, hortalizas feculentas, leche en polvo, carne ovina y porcina, aceites, grasas, azúcar de mesa y sal, y menos panificados y galletitas, yogur, quesos, manteca, dulces, golosinas, postres, gaseosas, jugos y comidas listas para

Tabla 2. Consumo aparente promedio de alimentos y bebidas por adulto equivalente, por quintil de ingreso y promedio, según entorno (g/d o ml/d). Argentina, 2004-2005.

\begin{tabular}{|c|c|c|c|c|c|c|c|c|c|c|c|c|c|c|}
\hline \multirow[t]{2}{*}{ Alimentos $\mathrm{y}$ bebidas } & \multicolumn{6}{|c|}{$\begin{array}{l}\text { Entorno urbano } \\
\text { (quintiles) }\end{array}$} & \multicolumn{6}{|c|}{$\begin{array}{c}\text { Entorno rural } \\
\text { (quintiles) }\end{array}$} & \multirow{2}{*}{$\begin{array}{l}\text { Diferencia } \\
\text { Rural- } \\
\text { urbano }\end{array}$} & \multirow{2}{*}{$\begin{array}{l}\text { Relación } \\
\text { Rural/ } \\
\text { urbano }\end{array}$} \\
\hline & 1 & 2 & 3 & 4 & 5 & $\mathrm{P}$ & 1 & 2 & 3 & 4 & 5 & $\mathrm{P}$ & & \\
\hline \multicolumn{15}{|l|}{ Cereales y legumbres } \\
\hline Arroz & 22,0 & 21,6 & 24,1 & 21,7 & 21,5 & 22,2 & 35,9 & 32,3 & 41,5 & 37,9 & 32,1 & 35,9 & 13,7 & 1,6 \\
\hline Harina de trigo & 23,9 & 19,5 & 20,7 & 17,3 & 14,3 & 19,1 & 153,5 & 122,2 & 91,9 & 77,6 & 92,3 & 117,7 & 98,7 & 6,2 \\
\hline Otros cereales (granos y harinas) & 7,1 & 8,4 & 9,8 & 9,9 & 9,7 & 9,0 & 26,4 & 42,7 & 18,5 & 22,8 & 41,8 & 30,0 & 21,0 & 3,3 \\
\hline Tapas frescas y masas & 2,1 & 4,3 & 6,3 & 7,8 & 8,7 & 5,9 & 0,2 & 0,8 & 1,5 & 2,5 & 1,7 & 1,1 & $-4,8$ & 0,2 \\
\hline Total cereales & 87,8 & 87,6 & 98,4 & 95,6 & 95,1 & 93,0 & 259,7 & 240,7 & 211,7 & 192,7 & 212,1 & 231,9 & 138,9 & 2,5 \\
\hline Legumbres frescas, secas y en conserva & 2,1 & 3,2 & 3,8 & 4,8 & 5,0 & 3,8 & 3,4 & 4,5 & 6,3 & 5,9 & 6,1 & 4,8 & 1,0 & 1,3 \\
\hline \multicolumn{15}{|l|}{ Panificados y galletitas } \\
\hline Pan de panadería & 161,6 & 162,8 & 150,7 & 132,1 & 106,0 & 142,2 & 126,7 & 157,7 & 170,7 & 213,8 & 202,6 & 162,8 & 20,7 & 1,1 \\
\hline Galletitas & 11,2 & 18,5 & 25,2 & 31,8 & 36,8 & 24,9 & 8,5 & 13,2 & 20,0 & 28,1 & 25,3 & 16,4 & $-8,6$ & 0,7 \\
\hline Total panificados y galletitas & 188,4 & 204,6 & 204,2 & 199,5 & 190,9 & 197,6 & 141,0 & 181,2 & 204,2 & 268,4 & 255,0 & 192,9 & $-4,7$ & 1,0 \\
\hline
\end{tabular}


Tabla 2. Continuación.

\begin{tabular}{|c|c|c|c|c|c|c|c|c|c|c|c|c|c|c|}
\hline \multirow[t]{2}{*}{ Alimentos y bebidas } & \multicolumn{6}{|c|}{$\begin{array}{l}\text { Entorno urbano } \\
\text { (quintiles) }\end{array}$} & \multicolumn{6}{|c|}{$\begin{array}{l}\text { Entorno rural } \\
\text { (quintiles) }\end{array}$} & \multirow{2}{*}{$\begin{array}{l}\text { Diferencia } \\
\text { Rural- } \\
\text { urbano }\end{array}$} & \multirow{2}{*}{$\begin{array}{c}\text { Relaciór } \\
\text { Rural/ } \\
\text { urbano }\end{array}$} \\
\hline & 1 & 2 & 3 & 4 & 5 & $\mathrm{P}$ & 1 & 2 & 3 & 4 & 5 & $\mathrm{P}$ & & \\
\hline \multicolumn{15}{|l|}{ Frutas } \\
\hline Banana & 10,2 & 13,6 & 19,9 & 22,3 & 23,8 & 21,8 & 7,8 & 9,4 & 9,2 & 17,0 & 15,4 & 15,5 & $-6,2$ & 0,7 \\
\hline Durazno & 2,0 & 4,0 & 6,0 & 7,3 & 8,4 & 20,2 & 0,7 & 1,4 & 1,7 & 12,3 & 9,9 & 16,4 & $-3,8$ & 0,8 \\
\hline Mandarina & 6,3 & 8,9 & 13,2 & 14,4 & 15,6 & 11,8 & 4,2 & 11,1 & 8,4 & 10,2 & 11,9 & 8,3 & $-3,5$ & 0,7 \\
\hline Manzana & 8,2 & 15,2 & 22,3 & 28,2 & 33,6 & 18,1 & 6,9 & 11,7 & 16,5 & 24,9 & 35,0 & 10,6 & $-7,5$ & 0,6 \\
\hline Naranja & 9,7 & 14,6 & 21,6 & 25,1 & 29,3 & 5,6 & 13,6 & 15,2 & 11,2 & 19,5 & 30,6 & 3,8 & $-1,8$ & 0,7 \\
\hline Pera & 1,2 & 2,5 & 5,0 & 6,5 & 7,8 & 4,7 & 0,3 & 1,9 & 1,7 & 3,3 & 1,9 & 1,5 & $-3,1$ & 0,3 \\
\hline Otras frutas frescas & 8,8 & 14,1 & 19,9 & 25,7 & 40,9 & 21,8 & 16,9 & 12,6 & 9,1 & 21,1 & 37,1 & 17,4 & $-4,4$ & 0,8 \\
\hline Frutas en conserva & 0,3 & 1,0 & 2,0 & 3,3 & 4,5 & 2,3 & 0,2 & 0,4 & 1,4 & 2,9 & 4,8 & 1,4 & $-0,9$ & 0,6 \\
\hline Futas secas y desecadas & 0,1 & 0,1 & 0,2 & 0,3 & 0,8 & 0,3 & 0,0 & 0,3 & 0,3 & 0,1 & 0,6 & 0,3 & $-0,1$ & 0,8 \\
\hline Total frutas & 46,9 & 74,1 & 110,0 & 133,3 & 164,7 & 106,6 & 50,7 & 64,0 & 59,4 & 111,4 & 147,0 & 75,3 & $-31,3$ & 0,7 \\
\hline \multicolumn{15}{|l|}{ Hortalizas } \\
\hline Papa & 87,1 & 96,6 & 102,2 & 97,2 & 76,4 & 91,8 & 97,7 & 97,6 & 114,9 & 98,9 & 136,7 & 105,3 & 13,4 & 1,1 \\
\hline Lechuga & 4,3 & 6,5 & 8,3 & 10,2 & 10,1 & 8,0 & 3,4 & 5,9 & 9,8 & 10,2 & 10,7 & 6,9 & $-1,1$ & 0,9 \\
\hline Tomate & 26,0 & 33,4 & 40,0 & 43,8 & 47,7 & 38,4 & 23,1 & 33,0 & 25,8 & 69,2 & 49,0 & 35,6 & $-2,8$ & 0,9 \\
\hline Zapallo & 6,5 & 9,1 & 12,6 & 11,9 & 14,4 & 11,0 & 12,6 & 12,3 & 10,4 & 13,3 & 12,8 & 12,3 & 1,3 & 1,1 \\
\hline Zanahoria & 9,6 & 11,8 & 14,5 & 14,7 & 15,6 & 13,3 & 12,2 & 14,7 & 15,8 & 24,8 & 16,3 & 15,7 & 2,4 & 1,2 \\
\hline Tomate en conserva & 12,7 & 13,8 & 15,6 & 15,9 & 15,6 & 14,8 & 10,7 & 11,9 & 19,6 & 18,8 & 19,5 & 14,7 & $-0,1$ & 1,0 \\
\hline Cebolla & 22,3 & 25,7 & 27,2 & 27,2 & 24,8 & 25,5 & 30,3 & 31,3 & 37,3 & 35,7 & 41,0 & 33,8 & 8,3 & 1,3 \\
\hline Otras hortalizas feculentas & 4,8 & 6,3 & 7,5 & 8,6 & 8,9 & 7,3 & 15,4 & 16,2 & 15,6 & 13,2 & 27,0 & 16,4 & 9,1 & 2,3 \\
\hline Otras hortalizas no feculentas & 20,0 & 29,7 & 39,0 & 47,6 & 61,8 & 32,7 & 23,4 & 31,3 & 32,7 & 40,7 & 41,7 & 15,1 & $-17,6$ & 0,5 \\
\hline Total hortalizas feculentas & 92,0 & 102,9 & 109,8 & 105,8 & 85,3 & 99,1 & 113,0 & 113,8 & 130,5 & 112,2 & 163,7 & 121,7 & 22,6 & 1,2 \\
\hline Total hortalizas no feculentas & 101,4 & 130,1 & 157,1 & 171,5 & 190,1 & 143,6 & 115,7 & 140,4 & 151,5 & 212,8 & 191,0 & 134,1 & $-9,5$ & 0,9 \\
\hline Total hortalizas & 193,3 & 232,9 & 266,9 & 277,3 & 275,4 & 242,7 & 228,7 & 254,1 & 282,0 & 325,0 & 354,8 & 255,7 & 13,0 & 1,1 \\
\hline Total hortalizas y frutas & 240,2 & 307,1 & 376,9 & 410,6 & 440,1 & 349,3 & 279,5 & 318,1 & 341,4 & 436,4 & 501,8 & 331,1 & $-18,3$ & 0,9 \\
\hline \multicolumn{15}{|l|}{ Lácteos } \\
\hline Leche & 60,2 & 95,2 & 131,6 & 148,8 & 163,0 & 120,8 & 49,2 & 82,8 & 118,2 & 176,3 & 153,1 & 99,3 & $-21,4$ & 0,8 \\
\hline Leche en polvo & 15,8 & 17,9 & 17,0 & 17,9 & 15,5 & 16,8 & 24,3 & 34,5 & 35,4 & 23,7 & 57,1 & 32,3 & 15,4 & 1,9 \\
\hline Yogur & 15,1 & 22,4 & 30,4 & 36,9 & 53,3 & 31,9 & 6,1 & 14,7 & 19,6 & 22,7 & 24,8 & 15,0 & $-17,0$ & 0,5 \\
\hline Quesos blandos & 5,5 & 10,4 & 14,0 & 18,5 & 21,9 & 14,2 & 2,7 & 5,1 & 7,1 & 8,2 & 24,3 & 7,4 & $-6,9$ & 0,5 \\
\hline Quesos duros & 1,0 & 1,8 & 2,9 & 3,2 & 4,8 & 2,8 & 0,7 & 0,7 & 1,6 & 2,8 & 3,2 & 1,5 & $-1,3$ & 0,5 \\
\hline Quesos semiduros & 1,2 & 2,0 & 2,9 & 4,4 & 6,9 & 3,5 & 0,8 & 1,6 & 0,8 & 4,1 & 7,1 & 2,2 & $-1,3$ & 0,6 \\
\hline Quesos crema y untable & 0,4 & 0,9 & 1,7 & 2,5 & 5,9 & 2,3 & 0,0 & 0,4 & 0,7 & 0,5 & 0,6 & 0,4 & $-1,9$ & 0,2 \\
\hline Postres lácteos y otros & 0,4 & 1,0 & 1,6 & 2,2 & 5,5 & 2,2 & 0,2 & 0,3 & 0,7 & 1,1 & 0,7 & 0,5 & $-1,7$ & 0,2 \\
\hline Total lácteos & 99,7 & 151,6 & 202,2 & 234,3 & 276,7 & 194,6 & 84,1 & 140,1 & 184,1 & 239,4 & 270,9 & 158,4 & $-36,1$ & 0,8 \\
\hline \multicolumn{15}{|l|}{ Carnes y huevo } \\
\hline Carne vacuna & 70,8 & 91,7 & 101,0 & 111,5 & 106,0 & 96,6 & 73,3 & 89,9 & 107,2 & 115,9 & 138,5 & 96,7 & 0,1 & 1,0 \\
\hline Pollo & 19,6 & 32,2 & 40,1 & 46,4 & 46,7 & 37,1 & 25,1 & 28,9 & 43,9 & 72,6 & 57,2 & 38,9 & 1,7 & 1,0 \\
\hline Pescados y mariscos & 2,6 & 4,5 & 7,0 & 10,5 & 13,9 & 7,8 & 2,5 & 2,5 & 6,1 & 5,3 & 3,2 & 3,6 & $-4,2$ & 0,5 \\
\hline Carne ovina y porcina & 1,9 & 2,2 & 2,5 & 2,3 & 4,0 & 2,6 & 6,0 & 25,5 & 16,5 & 21,8 & 16,2 & 22,4 & 19,8 & 8,6 \\
\hline Productos cárnicos semielaborados & 8,1 & 12,0 & 14,7 & 17,3 & 21,2 & 14,9 & 6,4 & 11,1 & 11,0 & 14,3 & 12,1 & 11,1 & $-3,8$ & 0,7 \\
\hline Vísceras, achuras y hueso & 15,5 & 14,4 & 11,9 & 10,1 & 8,6 & 12,0 & 17,9 & 17,5 & 16,0 & 18,1 & 9,0 & 16,5 & 4,4 & 1,4 \\
\hline Fiambres y embutidos & 9,1 & 13,1 & 15,8 & 18,3 & 21,7 & 15,7 & 8,9 & 16,8 & 15,6 & 19,2 & 17,9 & 14,4 & $-1,3$ & 0,9 \\
\hline Huevo & 15,4 & 18,8 & 21,5 & 22,2 & 23,7 & 20,4 & 16,8 & 23,0 & 31,6 & 33,9 & 34,6 & 25,3 & 4,9 & 1,2 \\
\hline Total carnes y huevo & 142,9 & 188,8 & 214,4 & 238,6 & 245,9 & 207,2 & 157,0 & 215,0 & 247,9 & 301,1 & 288,7 & 228,8 & 21,6 & 1,1 \\
\hline \multicolumn{15}{|l|}{ Aceites y grasas } \\
\hline Aceite mezcla & 12,2 & 13,9 & 16,7 & 14,6 & 13,8 & 14,3 & 19,5 & 19,1 & 34,5 & 27,0 & 18,1 & 22,9 & 8,6 & 1,6 \\
\hline Aceites puros & 6,2 & 10,7 & 12,5 & 16,9 & 19,2 & 13,2 & 12,4 & 22,0 & 19,3 & 19,1 & 25,6 & 18,3 & 5,0 & 1,4 \\
\hline Manteca & 1,1 & 1,9 & 2,4 & 3,4 & 3,8 & 2,6 & 0,5 & 0,8 & 2,0 & 2,0 & 0,9 & 1,1 & $-1,5$ & 0,4 \\
\hline Mayonesa & 1,8 & 2,7 & 3,3 & 4,0 & 4,7 & 3,3 & 0,9 & 1,1 & 3,7 & 5,7 & 4,4 & 2,5 & $-0,8$ & 0,8 \\
\hline Margarina y otras grasas & 2,9 & 2,6 & 3,3 & 3,4 & 4,7 & 3,4 & 13,5 & 10,1 & 7,5 & 5,7 & 11,4 & 10,3 & 6,9 & 3,0 \\
\hline Total aceites y grasas & 24,2 & 31,8 & 38,2 & 42,3 & 46,3 & 36,8 & 46,7 & 53,1 & 66,9 & 59,5 & 60,4 & 55,0 & 18,3 & 1,5 \\
\hline
\end{tabular}


Tabla 2. Continuación.

\begin{tabular}{|c|c|c|c|c|c|c|c|c|c|c|c|c|c|c|}
\hline \multirow[t]{2}{*}{ Alimentos y bebidas } & \multicolumn{6}{|c|}{$\begin{array}{l}\text { Entorno urbano } \\
\text { (quintiles) }\end{array}$} & \multicolumn{6}{|c|}{$\begin{array}{l}\text { Entorno rural } \\
\text { (quintiles) }\end{array}$} & \multirow{2}{*}{$\begin{array}{l}\text { Diferencia } \\
\text { Rural- } \\
\text { urbano }\end{array}$} & \multirow{2}{*}{$\begin{array}{l}\text { Relación } \\
\text { Rural/ } \\
\text { urbano }\end{array}$} \\
\hline & 1 & 2 & 3 & 4 & 5 & $\mathrm{P}$ & 1 & 2 & 3 & 4 & 5 & $\mathrm{P}$ & & \\
\hline \multicolumn{15}{|l|}{ Azúcares, dulces, golosinas y postres } \\
\hline Azúcar & 35,0 & 34,7 & 38,6 & 34,8 & 32,0 & 35,0 & 68,9 & 76,8 & 91,3 & 98,0 & 75,8 & 79,5 & 44,5 & 2,3 \\
\hline Dulces compactos y untables & 3,7 & 6,6 & 9,0 & 11,2 & 15,1 & 9,2 & 2,9 & 5,6 & 9,9 & 12,9 & 6,5 & 6,6 & $-2,7$ & 0,7 \\
\hline Helados y postres & 3,2 & 5,0 & 6,3 & 9,6 & 15,0 & 6,1 & 1,4 & 2,3 & 3,6 & 7,1 & 16,8 & 3,9 & $-2,1$ & 0,6 \\
\hline Chocolates y bombones & 0,7 & 1,2 & 1,9 & 2,4 & 4,3 & 1,9 & 0,3 & 0,6 & 0,8 & 2,4 & 2,4 & 0,7 & $-1,2$ & 0,4 \\
\hline Otras golosinas & 0,1 & 0,2 & 0,2 & 0,2 & 0,3 & 2,1 & 0,1 & 0,1 & 0,1 & 1,6 & 0,1 & 1,1 & $-1,0$ & 0,5 \\
\hline Cacao azucarado o no & 0,5 & 0,7 & 0,9 & 1,3 & 1,1 & 0,9 & 0,2 & 0,2 & 1,6 & 0,7 & 0,6 & 0,6 & $-0,3$ & 0,6 \\
\hline Total azúcares, dulces, golosinas y postres & 47,6 & 53,5 & 61,9 & 65,3 & 74,5 & 60,6 & 76,4 & 89,5 & 111,8 & 127,1 & 106,8 & 96,0 & 35,4 & 1,6 \\
\hline \multicolumn{15}{|l|}{ Bebidas sin alcohol } \\
\hline Otras bebidas sin alcohol & 9,1 & 14,2 & 21,5 & 31,6 & 50,8 & 25,8 & 2,7 & 7,0 & 13,6 & 16,3 & 31,9 & 10,9 & $-14,9$ & 0,4 \\
\hline \multicolumn{15}{|l|}{ Infusiones } \\
\hline Café & 29,4 & 59,9 & 122,1 & 144,3 & 273,7 & 127,9 & 17,7 & 38,1 & 105,7 & 97,2 & 84,4 & 56,6 & $-71,2$ & 0,4 \\
\hline $\mathrm{Te}$ & 34,6 & 46,0 & 75,4 & 76,2 & 96,2 & 66,3 & 32,5 & 37,6 & 92,5 & 81,0 & 43,5 & 52,1 & $-14,1$ & 0,8 \\
\hline Yerba mate & 241,0 & 305,4 & 388,3 & 419,5 & 442,7 & 361,4 & 395,0 & 476,1 & 743,2 & 723,5 & 951,8 & 585,9 & 224,5 & 1,6 \\
\hline Otras infusiones & 0,6 & 1,4 & 2,2 & 6,0 & 13,3 & 4,8 & 0,2 & 0,8 & 0,2 & 0,6 & 1,9 & 0,6 & $-4,2$ & 0,1 \\
\hline \multicolumn{15}{|l|}{ Bebidas con alcohol } \\
\hline Cerveza & 8,3 & 16,8 & 18,9 & 25,5 & 39,9 & 22,1 & 8,5 & 15,9 & 33,8 & 26,1 & 34,8 & 20,1 & $-2,0$ & 0,9 \\
\hline Vino & 14,5 & 24,0 & 34,3 & 45,5 & 62,1 & 36,5 & 25,5 & 57,3 & 61,3 & 83,5 & 95,7 & 55,5 & 19,0 & 1,5 \\
\hline Otras bebidas alcohólicas & 3,3 & 3,8 & 3,4 & 49,2 & 9,9 & 14,1 & 2,1 & 6,4 & 7,8 & 15,0 & 24,5 & 8,6 & $-5,5$ & 0,6 \\
\hline \multicolumn{15}{|l|}{ Comidas listas para consumir en el hogar } \\
\hline \multicolumn{15}{|l|}{ Otros alimentos } \\
\hline Sal de mesa y de cocina & 4,1 & 5,1 & 5,2 & 5,8 & 5,0 & 5,1 & 11,0 & 9,6 & 10,0 & 11,0 & 9,1 & 10,1 & 5,0 & 2,0 \\
\hline Salsas y aderezos & 0,1 & 0,2 & 0,3 & 0,5 & 0,9 & 0,4 & 0,0 & 0,0 & 0,1 & 0,6 & 0,0 & 0,1 & $-0,3$ & 0,3 \\
\hline Productos de copetín & 0,6 & 0,8 & 0,8 & 1,4 & 2,0 & 1,1 & 0,3 & 0,6 & 0,3 & 0,7 & 1,6 & 0,6 & $-0,6$ & 0,5 \\
\hline
\end{tabular}

comer que los urbanos del mismo quintil. En hogares urbanos del quintil 5 se consumen más pescados, mariscos y productos cárnicos semielaborados y menos huevos que en los hogares rurales del quintil 5 (Tabla 2).

La disponibilidad de energía es mayor en los hogares rurales, como también la proporción de energía proveniente de carbohidratos, alcohol, ácidos grasos omega 6 (n-6) y azúcares agregados. A pesar de las diferencias absolutas, al equiparar los valores y estimarlos cada $1.000 \mathrm{kcal}$, los hogares rurales solo presentan mayor disponibilidad de sodio, folato y vitamina $\mathrm{E}$; mientras que los hogares urbanos muestran valores superiores de colesterol, vitamina A, C, B12 y niacina, cada $1.000 \mathrm{kcal}$. La proporción de energía aportada por macronutrientes aumenta a 
Tabla 3. Ingesta aparente de energía y nutrientes por adulto equivalente, por quintil de ingreso y promedio, según entorno. Argentina, 2004-2005.

\begin{tabular}{|c|c|c|c|c|c|c|c|c|c|c|c|c|}
\hline \multirow[t]{2}{*}{ Nutrientes } & \multicolumn{6}{|c|}{$\begin{array}{l}\text { Entorno urbano } \\
\text { (quintiles) }\end{array}$} & \multicolumn{6}{|c|}{$\begin{array}{l}\text { Entorno rural } \\
\text { (quintiles) }\end{array}$} \\
\hline & 1 & 2 & 3 & 4 & 5 & Promedio & 1 & 2 & 3 & 4 & 5 & Promedio \\
\hline Energía (Kcal) & 1.867 & 2.232 & 2.542 & 2.773 & 3.071 & 2.511 & 2.683 & 3.089 & 3.400 & 3.790 & 3.800 & 3.194 \\
\hline Carbohidratos (\% energía) & 59,9 & 56,0 & 54,3 & 51,7 & 49,6 & 53,6 & 61,8 & 58,7 & 56,1 & 55,4 & 54,9 & 58,0 \\
\hline Proteínas (\% energía) & 13,7 & 14,3 & 14,3 & 14,7 & 14,7 & 14,3 & 11,7 & 12,3 & 12,3 & 14,0 & 13,9 & 12,7 \\
\hline Grasas (\% energía) & 25,3 & 28,3 & 29,9 & 31,3 & 33,2 & 30,3 & 24,7 & 27,0 & 29,3 & 28,0 & 28,2 & 27,2 \\
\hline Alcohol (\% energía) & 0,8 & 1,1 & 1,3 & 2,1 & 2,2 & 1,6 & 0,8 & 1,7 & 1,8 & 2,3 & 2,5 & 1,7 \\
\hline Azúcares totales (\% energía) & 17,9 & 18,3 & 18,8 & 19,2 & 20,1 & 19,0 & 16,3 & 17,7 & 19,0 & 19,8 & 17,6 & 17,9 \\
\hline Azúcares agregados (\% energía) & 13,7 & 13,5 & 13,5 & 13,3 & 14,0 & 13,6 & 13,3 & 14,3 & 15,4 & 15,5 & 12,7 & 14,2 \\
\hline AGS (\% energía) & 8,0 & 9,1 & 9,7 & 10,4 & 11,3 & 10,0 & 7,2 & 7,6 & 8,0 & 8,3 & 8,9 & 7,9 \\
\hline AGMI (\% energía) & 8,3 & 9,1 & 9,6 & 10,0 & 10,7 & 9,8 & 8,1 & 8,6 & 9,3 & 9,0 & 9,0 & 8,7 \\
\hline AGPI n3 (\% energía) & 0,3 & 0,3 & 0,4 & 0,4 & 0,4 & 0,3 & 0,3 & 0,3 & 0,4 & 0,3 & 0,3 & 0,3 \\
\hline AGPI n6 (\% energía) & 6,8 & 7,6 & 7,8 & 7,9 & 7,7 & 7,6 & 7,7 & 8,7 & 9,8 & 8,1 & 8,2 & 8,4 \\
\hline Grasas Trans (\% energía) & 0,5 & 0,6 & 0,7 & 0,7 & 0,7 & 0,7 & 0,5 & 0,4 & 0,4 & 0,5 & 0,5 & 0,5 \\
\hline Colesterol (mg) & 199 & 263 & 307 & 354 & 408 & 308 & 203 & 272 & 341 & 439 & 426 & 303 \\
\hline Fibra (g) & 12,5 & 14,6 & 16,6 & 17,8 & 19,0 & 16,2 & 17,0 & 19,8 & 18,6 & 21,9 & 24,7 & 19,5 \\
\hline Sodio (mg) & 3.191 & 3.925 & 4.224 & 4.722 & 4.729 & 4.174 & 5.585 & 5.483 & 5.953 & 6.948 & 6.243 & 6.047 \\
\hline Calcio (mg) & 335 & 483 & 622 & 744 & 918 & 625 & 320 & 454 & 555 & 727 & 878 & 517 \\
\hline Hierro (mg) & 13,4 & 15,5 & 16,9 & 17,9 & 18,7 & 16,5 & 18,5 & 20,5 & 21,0 & 24,6 & 25,9 & 21,1 \\
\hline Zinc (mg) & 8,7 & 10,7 & 11,9 & 13,0 & 14,1 & 11,8 & 11,0 & 13,6 & 14,0 & 16,6 & 17,6 & 13,7 \\
\hline Vitamina A (ug ER) & 725 & 937 & 1132 & 1241 & 1429 & 1099 & 732 & 873 & 926 & 1341 & 1413 & 964 \\
\hline Vitamina C (mg) & 38,6 & 51,9 & 64,4 & 73,4 & 88,8 & 63,9 & 40,2 & 51,0 & 49,9 & 68,2 & 76,0 & 52,6 \\
\hline Folato (ug FDE) & 755 & 800 & 824 & 812 & 802 & 799 & 1.114 & 1.134 & 1.108 & 1.283 & 1.286 & 1.162 \\
\hline Vitamina B12 (ug) & 4,5 & 5,3 & 5,6 & 5,9 & 6,0 & 5,5 & 4,0 & 4,4 & 4,7 & 6,0 & 5,0 & 4,6 \\
\hline Tiamina (mg) & 1,9 & 2,1 & 2,2 & 2,2 & 2,3 & 2,1 & 2,6 & 3,0 & 2,8 & 3,6 & 3,4 & 3,0 \\
\hline Riboflavina (mg) & 1,6 & 1,9 & 2,1 & 2,3 & 2,5 & 2,1 & 1,7 & 2,1 & 2,3 & 3,0 & 2,9 & 2,2 \\
\hline Niacina (mg) & 16,9 & 20,4 & 22,6 & 24,7 & 26,3 & 22,1 & 18,9 & 23,5 & 24,9 & 33,1 & 32,1 & 24,5 \\
\hline Vitamina E (mg) & 13,2 & 17,0 & 20,4 & 21,4 & 22,9 & 19,1 & 21,0 & 24,7 & 35,5 & 31,5 & 28,9 & 26,7 \\
\hline Vitamina D (ug) & 1,3 & 1,9 & 2,4 & 2,7 & 3,2 & 2,3 & 1,3 & 2,1 & 2,5 & 3,5 & 3,3 & 2,2 \\
\hline
\end{tabular}

medida que aumentan los ingresos del hogar, a excepción de los carbohidratos, que descienden al crecer el nivel de ingreso tanto en hogares urbanos como en rurales. Los ácidos grasos omega 3 (n-3), las grasas trans, y los folatos no muestran tendencia según el ingreso del hogar; mientras que los azúcares se mantienen estables en hogares urbanos con diferente nivel de ingreso y dibujan una $U$ invertida en los hogares rurales, con valores máximos en los quintiles 3 y 4 (Tabla 3).

\section{DISCUSION}

El presente trabajo tuvo por objetivo describir el consumo aparente de alimentos y la disponibilidad de energía y nutrientes de hogares urbanos y rurales de la Argentina, según su nivel de ingreso. La alimentación constituye un componente central de varios de los indicadores de bienestar que se utilizan para evaluar seguridad alimentaria, nutrición, salud y pobreza, dado que representa, 
aproximadamente, entre un tercio y la mitad del presupuesto de los hogares ${ }^{(28,29,30)}$. Es de gran importancia conocer sus características para realizar un mejor monitoreo y planificación de políticas alimentarias y sociales. Por escasez de datos de consumo alimentario con representatividad nacional, las encuestas de gastos de hogares proporcionan una fuente promisoria de información para la planificación y la formulación de políti$\mathrm{cas}^{(31)}$. Estas encuestas han pasado de realizarse en apenas 22 países en $1950^{(32)}$ a más de 100 en la actualidad ${ }^{(33)}$, incluso con información seriada y, en muchos casos, con muestras en entornos urbanos y rurales. Al utilizar la información recopilada por estas encuestas, es necesario conocer, por un lado, sus limitaciones intrínsecas, como la ausencia de datos sobre la distribución de alimentos dentro del hogar y la falta de registro de desperdicios de alimentos o aquellos destinados para consumo animal, la falta de registro de alimentos que se reciben por programas sociales o gratuitamente; $y$, por otro, sus ventajas, como la representatividad, periodicidad y la forma no intrusiva de obtener información alimentaria en los hogares ${ }^{(16)} y$, particularmente, en la Argentina por ser la única fuente de información sobre consumo de alimentos de población rural con representatividad nacional.

Dado que se trata de un análisis sobre población denominada rural, es necesario aclarar que no existe una definición universal de entorno rural ni mucho menos definiciones oficiales compartidas por todos los países, ni siquiera los de una misma región o bloque de países. Las definiciones utilizadas varían de manera significativa entre países, ya sea porque se prefieren alternativamente criterios estadísticos o geográficos, o simplemente porque los límites cuantitativos, siempre arbitrarios, difieren de un país a otro. En la mayoría de los casos, rural corresponde a una categoría definida por defecto (lo que no es urbano), sin aludir a características propias $^{(2,34)}$. Por lo que las comparaciones entre países y regiones resultan difíciles de realizar $y$, en caso de existir información, deben realizarse con tal consideración.
En primer lugar, este trabajo evidencia las disparidades sociodemográficas de los hogares urbanos y rurales de la Argentina, reflejado en la cobertura de salud, el nivel educativo y los ingresos del hogar, situación que se repite en la mayoría de los países de Latinoamérica, y que ha sido bien documentada, por su repercusión en las condiciones de vida y de salud de la población ${ }^{(1)}$; como así también la distribución heterogénea de los hogares rurales en el país, alcanzando a casi uno de cada cinco hogares en el norte y apenas uno de cada veinte en el sur de país.

En segundo lugar, del análisis de los datos surge que, además de las disparidades sociodemográficas, existen marcadas diferencias en el patrón de consumo de alimentos y bebidas en los hogares rurales y urbanos de la Argentina, que se suman a las diferencias asociadas a los ingresos del hogar. El consumo de alimentos se ve afectado de manera variable por una amplia gama de factores que incluyen la disponibilidad, la accesibilidad alimentaria y la elección de alimentos que, a su vez, pueden estar influenciados por la ubicación geográfica, las condiciones demográficas, el nivel de ingresos, el nivel socioeconómico, la urbanización, la globalización, la comercialización, la religión, la cultura y las actitudes del consumidor ${ }^{(35)}$. En Argentina, los hogares rurales presentan mayor consumo aparente de gran parte de los grupos de alimentos. Posiblemente estas diferencias observadas se relacionen con las condiciones en la disponibilidad y la accesibilidad de algunos grupos de alimentos en el entorno rural como, por ejemplo, el caso de los alimentos frescos de más difícil acceso, y la carne ovina y porcina que, por ser muchas veces de producción local, se consume en mayor cantidad. También existen diferentes costumbres en la adquisición y la elaboración de alimentos en ambos entornos, ejemplos clave son el mayor consumo aparente de harina de trigo, margarina, grasas animales, azúcar de mesa y sal ligados a la mayor elaboración doméstica de alimentos en el entorno rural, mientras que el mayor consumo de pastas y masas frescas, de productos cárnicos semielaborados y de comidas listas 
en el urbano denota la búsqueda de practicidad a la hora de cocinar. La vida en entornos urbanos se asocia a mayor comercialización, más acceso a los medios de comunicación modernos y más infraestructura de distribución de alimentos ${ }^{(36)}$, esto finalmente facilita y favorece la globalización de los patrones de consumo ${ }^{(35)}$; aunque estos patrones también han comenzado a vislumbrase en áreas rurales de varias regiones del mundo ${ }^{(37)}$.

Con relación al nivel de ingreso, este análisis permite identificar dos escenarios. Por un lado, al comparar los hogares urbanos y rurales del mismo nivel de ingresos, se observan diferencias en el consumo de alimentos que parecerían responder a un patrón de consumo asociado más con el entorno que con el nivel de ingresos, especialmente, en los hogares de menores ingresos. Por otro lado, se observa que, con el incremento de los ingresos, los hogares tanto urbanos como rurales progresan a una dieta más variada y económicamente más cara, aumenta la cantidad y la diversidad de frutas y hortalizas no feculentas, el consumo de lácteos, especialmente los quesos que en los hogares urbanos cuadriplican su consumo entre el primer y el último quintil, y en los rurales se consumen casi nueve veces más en los hogares de mayores ingresos en relación con los de más bajos ingresos. En la mayoría de los países industrializados, los efectos del aumento de los ingresos, generalmente, se han considerado beneficiosos, lo que resulta en dietas de mejor calidad, mejores cuidados de la salud, menor morbilidad y mortalidad por enfermedades infecciosas y menor riesgo de obesidad $^{(38,39)}$.

La mayor presencia de huertas y corrales en el entorno rural podría estar asociado al mayor consumo aparente de carne ovina y porcina, huevo, pollo, pero es destacable el menor consumo de hortalizas y frutas de los hogares rurales, lo que plantea el desafío de reforzar las estrategias que mejoren la incorporación en la dieta familiar de los alimentos obtenidos por autoproducción en la huerta(40).
En la mayoría de los países, la falta de datos de consumo de alimentos, especialmente datos representativos a nivel nacional, ha obstaculizado la comprensión de las ingestas y patrones alimentarios, en particular, en los grupos poblacionales con diferentes condiciones sociodemográficas o de distintas etnias, lo que a su vez ha paralizado el diseño, monitoreo y evaluación de los programas de alimentación y nutrición ${ }^{(31)}$. Carecer de información acerca de los patrones de consumo de alimentos de la población es un obstáculo para la evaluación de la efectividad e impacto de diversas intervenciones alimentarias ${ }^{(41)}$. Es por eso que es necesario identificar fuentes de datos sobre consumo de alimentos que puedan brindar información al respecto. A pesar de las deficiencias propias, las encuestas de consumo y gastos de los hogares se utilizan cada vez más para abordar la brecha de información alimentaria y nutricional ${ }^{(42)}$.

Los resultados que arroja este trabajo deben ser interpretados con la consideración de que la encuesta fue realizada en un período posterior a la crisis económica, política y social que afectó a Argentina desde 2001, cuando el $60,3 \%$ de los hogares urbanos con niños estaban por debajo del umbral de pobreza, alcanzando el $77,2 \%$ en la región noreste y el $75,7 \%$ en el noroeste, y el $35 \%$ con necesidades básicas insatisfechas ${ }^{(22)}$.

Este análisis evidencia que existe un patrón de consumo aparente de alimentos y bebidas distinto entre hogares rurales y urbanos de Argentina, y además existen diferencias entre los hogares según el nivel de ingresos en ambos entornos. La identificación del factor que más influye en el consumo de alimentos es un aspecto que escapa a este trabajo, pero que sería de gran interés para futuras investigaciones.

Conocer el consumo de alimentos y la ingesta de nutrientes es esencial para comprender la alimentación poblacional, monitorear su calidad e informar las políticas alimentarias y nutricionales ${ }^{(31)}$, y poder cuantificar las diferencias y su magnitud es de gran utilidad para buscar soluciones acordes a cada grupo. 


\section{REFERENCIAS BIBLIOGRÁFICAS}

1. United Nations, Department of Economic and Social Affairs, Population Division. World urbanization prospects: The 2014 revision [Internet]. New York: United Nations; 2014 [citado 12 feb 2019]. Disponible en: https://tinyurl.com/y9uunl8u.

2. Castro H, Reboratti C. Revisión del concepto de ruralidad en la Argentina y alternativas posibles para su redefinición. Buenos Aires: Secretaría Agricultura, Ganadería, Pesca y Alimentos; 2007.

3. Instituto Nacional de Estadísticas y Censos. Población e inclusión social en la Argentina del Bicentenario: Indicadores demográficos y sociales [Internet]. Ciudad Autónoma de Buenos Aires: INDEC; 2015 [citado 12 feb 2019]. Disponible en: https://tinyurl.com/y3oconkv.

4. Instituto Nacional de Estadísticas y Censos. Censo Nacional de Población y Vivienda, 1991 [Internet]. 1991 [citado 12 feb 2019]. Disponible en: https://tinyurl.com/y2xb5r9j.

5. Instituto Nacional de Estadísticas y Censos. Censo Nacional de Población, Hogares y Viviendas, 2010 [Internet]. 2010 [citado 12 feb 2019]. Disponible en: https://tinyurl.com/yy4kpdmk.

6. Brug J. Determinants of healthy eating: motivation, abilities and environmental opportunities. Family Practice. 2008;25(Suppl 1):i50-i55. doi: 10.1093/fampra/cmn063.

7. Tucker KL, Buranapin S. Nutrition and aging in developing countries. Journal of Nutrition. 2001; 131(9):2417S-2423S. doi: 10.1093/jn/131.9.2417S.

8. Popkin BM, Siega-Riz AM, Haines PS. A comparison of dietary trends among racial and socioeconomic groups in the United States. New England Journal of Medicine. 1996;335(10):716-720. doi: 10.1056/NEJM199609053351006.

9. Popkin BM, Haines PS, Siega-Riz AM. Dietary patterns and trends in the United States: the UNC-CH approach. Appetite. 1999;32(1):8-14. doi: 10.1006/appe.1998.0190.

10. Popkin BM. Nutrition in transition: the changing global nutrition challenge. Asia Pacific Journal of Clinical Nutrition. 2001;10(Suppl):S13-S18.

11. Lytle LA. Measuring the food environment: state of the science. American Journal of Preventive Medicine. 2009;36(Suppl 4):S134-S144. doi: 10.1016/j.amepre.2009.01.018.

12. Glanz K. Measuring food environments: a historical perspective. American Journal of Pre- ventive Medicine. 2009;36(4 Suppl):S93-S98. doi: 10.1016/j.amepre.2009.01.010.

13. McLeroy KR, Bibeau D, Steckler A, Glanz K. An ecological perspective on health promotion programs. Health Education Quarterly. 1988;15(4):351377.

14. Rideout K, Mah C, Minaker L. Food environments: An introduction for public health practice [Internet]. Vancouver: National Collaborating Centre for Environmental Health; 2015 [citado 12 feb 2019]. Disponible en: https://tinyurl.com/ y2hy4n7j.

15. Glanz K, Sallis JF, Saelens BE, Frank LD. Healthy nutrition environments: concepts and measures. American Journal of Health Promotion. 2005;19(5):330-333. doi: 10.4278/0890-117119.5.330.

16. Fiedler JL, Lividini K, Bermudez OI, Smitz MF. Household consumption and expenditures surveys (HCES): a primer for food and nutrition analysts in low- and middle-income countries. Food and Nutrition Bulletin. 2012;33(Suppl 3):S170-S184. doi: $10.1177 / 15648265120333$ S205.

17. Fiedler JL, Smitz MF, Dupriez O, Friedman J. Household income and expenditure surveys: a tool for accelerating the development of evidence-based fortification programs. Food and Nutrition Bulletin. 2008;29(4):306-319. doi: $10.1177 / 156482650802900407$.

18. Naska A, Vasdekis VG, Trichopoulou A. A preliminary assessment of the use of household budget survey data for the prediction of individual food consumption. Public Health Nutrition. 2001;4(5B):1159-1165.

19. Instituto Nacional de Estadísticas y Censos. El gasto de consumo de los hogares urbanos en la Argentina: Un análisis a partir de las mediciones de 1996/1997, 2004/2005 y 2012/2013 [Internet]. Ciudad Autónoma de Buenos Aires: INDEC; 2014 [citado 12 feb 2019]. Disponible en: https:// tinyurl.com/y5nxpz5t.

20. Instituto Nacional de Estadísticas y Censos. Encuesta Nacional de Gastos de los Hogares 2004-2005 [Internet]. 2007 [citado 12 feb 2019]. Disponible en: https://tinyurl.com/yyc85bv5.

21. Instituto de Nutrición de Centro América y Panamá. Consumo Aparente 2019. INCAP [Internet]; c2019 [citado 12 feb 2019]. Disponible en: https://tinyurl.com/y35fz25p.

22. Ministerio de Salud de la Nación. Encuesta Nacional de Nutrición y Salud: Documento de re- 
sultados 2007 [Internet]. Buenos Aires: Ministerio de Salud de la Nación; 2007 [citado 12 feb 2019]. Disponible en: https://tinyurl.com/y5xotrpr.

23. López LB, Suárez MM. Alimentación saludable: Guía práctica para su realización. Buenos Aires: Akadia; 2011.

24. Ministerio de Salud de la Nación. Guías alimentarias para la población argentina: Documento técnico metodológico [Internet]. Buenos Aires: Ministerio de Salud de la Nación; 2016 [citado 12 feb 2019]. Disponible en: https://tinyurl.com/ y25r857j.

25. Lema S, Longo EN, Lopresti A. Guías alimentarias para la población argentina: manual de multiplicadores [Internet]. Buenos Aires: Asociación Argentina de Dietistas y Nutricionistas Dietistas; 2003 [citado 12 feb 2019]. Disponible en: https:// tinyurl.com/y6tyozvs.

26. Universidad Nacional de Luján. Tablas de composición de alimentos [Internet]. c2010 [citado 12 feb 2019]. Disponible en: https://tinyurl.com/ yxma7zmq.

27. United States Department of Agriculture, Agricultural Research Service. USDA National Nutrient Database for Standard Reference [Internet]. United States Department of Agriculture; 2016 [citado 12 feb 2019]. Disponible en: https:// tinyurl.com/y7d6dgkk.

28. Borlizzi A, Delgrossi MA, Cafiero C. National food security assessment through the analysis of food consumption data from household consumption and expenditure surveys: The case of Brazil's pesquisa de orçamento familiares 2008/09. Food Policy. 2017;72(C):20-26. doi: 10.1016/j.foodpol.2017.08.009.

29. Backiny-Yetna P, Steele D, Yacoubou Djima I. The impact of household food consumption data collection methods on poverty and inequality measures in Niger. Food Policy. 2017;72(C):7-19. doi: 10.1016/j.foodpol.2017.08.008.

30. United States Department of Agriculture, Economic Research Service. International food consumption patterns, 2005 [Internet]. United States Department of Agriculture; 2005 [citado 12 feb 2019]. Disponible en: https://tinyurl.com/ y53wchzz.

31. Sununtnasuk C, Fiedler JL. Can householdbased food consumption surveys be used to make inferences about nutrient intakes and inadequacies?: A Bangladesh case study. Food Policy. 2017;72(C):121-131. doi: 10.1016/j.foodpol.2017. 08.018 .
32. Jolliffe $D$, Lanjouw $P$, Chen $S$, Kraay A, Meyer C, Negre $M$, et al. A measured approach to ending poverty and boosting shared prosperity: Concepts, data, and the twin goals [Internet]. Washington DC: The World Bank; 2015 [citado 12 feb 2019]. Disponible en: https://tinyurl.com/kgwznb2.

33. Ferreira FHG, Chen S, Dabalen A, Dikhanov Y, Hamadeh N, Jollif D, Narayan A, et al. A global count of the extreme poor in 2012: data issues, methodology and initial results: Policy research working paper [Internet]. Washington DC: The World Bank; 2015 [citado 12 feb 2019]. Disponible en: https://tinyurl.com/y26crdyc.

34. Siqueira D, Osório R. O conceito de Rural. En: Giarracca N, (ed.). ¿Una nueva ruralidad en América Latina? Buenos Aires: CLACSO; 2001. p. 67-79.

35. KearneyJ. Food consumption trends and drivers. Philosophical Transactions of the Royal Society B, Biological Sciences. 2010;365(1554):2793-2807. doi: 10.1098/rstb.2010.0149.

36. Hawkes C. Uneven dietary development: linking the policies and processes of globalization with the nutrition transition, obesity and diet-related chronic diseases. Globalization and Health. 2006;2:4. doi: 10.1186/1744-8603-2-4.

37. Walker RE, Keane CR, Burke JG. Disparities and access to healthy food in the United States: A review of food deserts literature. Health \& Place. 2010;16(5):876-884. doi: 10.1016/j.healthplace.2010.04.013.

38. Marmot M. Income inequality, social environment, and inequalities in health. Journal of Policy Analysis and Management. 2001;20(1):156-159. doi: 10.1002/1520-6688(200124)20:1 < 156::AIDPAM2009>3.0.CO;2-9.

39. Marmot M. The influence of income on health: views of an epidemiologist. Health Affairs. 2002;21(2):31-46.

40. Organización de las Naciones Unidas para la Agricultura y la Alimentación. De la huerta a la mesa: Promoción del consumo de frutas y vegetales a partir de huertas familiares [Internet]. Santiago de Chile: Organización de las Naciones Unidas para la Agricultura y la Alimentación; 2011 [citado 12 feb 2019]. Disponible en: https:// tinyurl.com/y6mmwvrt.

41. Neufeld LM, Tolentino L. Nutritional surveillance: developing countries. En: Allen LH, Prentice A, Caballero B, (eds.). Encyclopedia of Human Nutrition. 3a ed. Oxford: Academic Press; 2013. p. 289-302. 
42. Fiedler JL. Towards overcoming the food consumption information gap: strengthening household consumption and expenditures surveys for food and nutrition policymaking. Global Food Security. 2013;2(1):56-63. doi: 10.1016/j. gfs.2012.09.002.

FORMA DE CITAR

Zapata ME, Rovirosa A, Carmuega E. Urbano y rural: diferencias en la alimentación de los hogares argentinos según nivel de ingreso y área de residencia. Salud Colectiva. 2019;15:e2201. doi: 10.18294/sc.2019.2201.

Recibido: 22 de febrero de 2019 | Versión final: 5 de junio de 2019 | Aprobado: 18 de junio de 2019

Esta obra está bajo una licencia de Creative Commons Reconocimiento-NoComercial 4.0

Internacional. Reconocimiento - Permite copiar, distribuir y comunicar públicamente la obra.

A cambio, se debe reconocer y citar al autor original. No Comercial — Esta obra no puede ser

utilizada con finalidades comerciales, a menos que se obtenga el permiso. 